\title{
Chronique d'une recherche: Réflexions sur l'étude interdisciplinaire d'un système agraire de la Montagne corse.
}

Jean-Pierre DEFFONTAINES, Camille RAICHON \& Bernard De VERNEUIL

I.N.R.A., Unité de Recherches sur les systèmes agraires et le développement de Versailles-Dijon (S.A.D.), Centre de Recherches agronomiques, Route de St-Cyr, F 78000 Versailles.

\section{RÉSUMÉ}

Interdisciplinarité, Pratique de recherche, Système agraire.

\begin{abstract}
A la fin d'une recherche interdisciplinaire qui a porté sur les systèmes agraires de la Montagne corse, les auteurs tentent de faire le bilan d'un travail de quatre années. Sous la forme d'une chronique, ils présentent à la fois les résultats et unc réflexion sur la démarche suivie.

Après avoir rappelé l'origine et les objectifs de la recherche, ils discutent des différents niveaux d'investigation retenus. Ces derniers permettent d'indiquer chronologiquement, sur une grille, la succession des actions et des événements marquants qui ont orienté leur travail vers une analyse des élevages de Castagniccia. Des remarques générales portent ensuite sur la démarche suivie. Enfin, les travaux et publications essentiels issus de cette recherche sont inventoriés.
\end{abstract}

\section{SUMMARY}

Interdisciplinarity,

Agrarian system,

Research practice.
Reflections on a research chronicle: An interdisciplinary study of agrarian system in the Corsican mountains.

The authors summarize four years of interdisciplinary research on agrarian systems in the Corsican mountains. The results are chronicled together with reflections on the procedures used. After presentation of the origin and aims of the programme, the different levels of investigation chosen are reviewed. The succession of actions and events which orientated the work towards an analysis of stock rearing in Castagniccia can be summarized chronologically in a diagram. The methods of investigation are considered in general terms, and finally the materials and publications emerging from the research are listed.
Les conséquences de l'exode agricole dans les régions défavorisées et surtout les zones de montagnes posent depuis de nombreuses années des problèmes sérieux à la collectivité. Pour mieux les connaître et leur apporter des solutions, quelques équipes de recherche, très isolées au départ, puis aidées par les pouvoirs publics et particulièrement par la D.G.R.S.T., ont engagé des études dans diverses régions. La nature des travaux à entreprendre a le plus souvent nécessité la constitution d'équipes de recherches interdisciplinaires regroupant des biologistes et des socio-économistes.

Devant la nouveauté à la fois du sujet d'études et des relations entre ces disciplines, les méthodes et les démarches de recherches étaient à inventer. Chaque équipe, selon sa composition et ses objectifs particuliers a ainsi mené des expériences epistémologiques. Cependant si les résultats des différents travaux ont été publiés, le plus souvent les méthodes utilisées ne sont pas ou peu connues. Devant la multiplication de ce type de recherche, il devient indispensable d'engager un débat à la fois sur la clarification de leurs objectifs, les méthodes les mieux appropriées et les intérêts et limites de l'interdisciplinarité. Cet article, qui relate la démarche suivie par une équipe de recherche du département de recherches sur les systèmes agraires et le développement de l'I.N.R.A., voudrait apporter une contribution à cette discussion.

\section{ORIGINE DE LA RECHERCHE}

Début 1974, une demande émanant de plusieurs élus de la Corse est adressée à la Direction de l'I.N.R.A. Elle souhaite que les préoccupations des différents organismes de recherche déjà présents dans l'île s'étendent aux problèmes posés par l'avenir de l'agriculture dans les zones de montagne. Une convention est signée entre le Préfet de Région et l'I.N.R.A. en mars 1974. A la suite d'une reconnaissance par quelques uns de ses chercheurs, l'ancien Service d'Expérimentation et d'Information de l'I.N.R.A. (S.E.I.) est chargé de répondre à cette demande.

Un groupe de travail est alors constitué. Au départ il réunit quatre agronomes, dont un corse, seul permanent sur 
place, un économiste et un sociologue du S.E.I., plus un sociologue enseignant à l'Institut National Agronomique Paris-Grignon (I.N.A.P.G.). Il se propose alors « d'examiner les possibilités d'évolution des activités agricoles dans diverses zones de la Corse intérieure en vue du maintien et de l'installation d'agriculteurs, tout en assurant une maîtrise du territoire ». Ce projet s'inscrit ainsi dans la problématique de l'équipe d'analyse de situation et de diagnostic régional qui, depuis plusieurs années, avec la collaboration de chercheurs d'autres disciplines de l'I.N.R.A. et d'enseignants se préoccupe des voies possibles de développement de l'agriculture dans le cadre de diverses régions: Millevaches, Vivarais, Jura, Aunis, Vosges.

Après discussion avec divers organismes publics et professionnels locaux, la recherche est envisagée sur trois régions très différentes: la Castagniccia au Nord-Est, le Niolu au Centre et l'Alta Rocca au Sud de l'île. Mais les difficultés d'accès et de déplacements amènent l'équipe à se limiter dans un premier temps à la Castagniccia qui cst aussi la région la mieux connue de l'agronome originaire de l'île.

En avril 1975, cette recherche fait l'objet d'une aide de la Délégation Générale à la Recherche Scientifique et Technique (D.G.R.S.T.), sous le titre : «Etude interdisciplinaire des systèmes agraires dans la Montagne corse. Eléments pour une stratégie de développement ».

\section{FORMULATION SCHÉMATIQUE DU PROBLEME DE RECHERCHE}

De climat méditerranéen, la Castagniccia fait partic du massif schisteux du Nord Est de la Corse. Elle est délimitée au Nord par le Golu et au Sud par le Tavignanu. Dynamique jusqu'au début du $20^{\circ}$ siècle, l'ancien système de production horto-pastoral et arboricole intensif a modelé un paysage qui apparaît encore aujourd'hui, malgré la disparition de la quasi-totalité des activités agricoles due au départ de la plupart des résidents permanents. Actuellement seul un élevage extensif utilise la végétation arbustive qui recouvre uniformément les anciennes occupations du territoire. La vie sociale s'éteignant, les mises en valeur antérieures comme les terrasses, les chemins, les sources se dégradent. De plus, la châtaigneraie, les productions originales de qualité, les capacités d'accueil, mais aussi les coutumes et les connaissances séculaires qui composent le patrimoine économique et culturel de la région disparaissent.

L'absence de politique de développement de l'agriculture de la montagne corse réduit tout espoir de changement dans cette évolution. Or, les conséquences à terme peuvent être graves et difficilement réversibles. Une telle évolution n'est pas acceptable par la population locale et les habitants de l'île, mais elle ne l'est pas non plus pour la société globale dont l'intérêt ne peut se limiter à regarder, indifférente, la disparition d'une population et l'abandon du territoire qui la fait vivre. Représentant un stade avancé sinon extrême de la régression démographique en milieu rural, le cas de la Castagniccia est fréquent dans les zones montagneuses du Bassin méditerranéen, si bien que répondre à la question : quelle stratégie de développement pour la Castagniccia? dépasse le cadre de la région étudiée.

Volontairement, notre travail se plaçait d'emblée dans une perspective définie de développement, celle de revivifier cette zone en y maintenant et en y attirant des hommes. Il s'appuyait sur deux hypothèses : d'une part la perspective de rénovation sociale envisagée impliquait le développe- ment des activités agricoles, mais également des activités non agricoles ; d'autre part elle n'était possible qu'avec une participation active des personnes et organismes ayant des projets ou des intérêts en Castagniccia. Tel était schématiquement le point de départ de la recherche en 1974.

\section{LES NIVEAUX DE RECHERCHE}

Les travaux précédents de l'équipe de recherche I.N.R.A.-E.N.S.S.A.A., notamment ceux réalisés dans la région de Rambervillers dans le département des Vosges (Groupe de recherches I.N.R.A.-E.N.S.S.A.A., 1973), ont montré l'intérêt d'étudier les projets des agriculteurs et de leurs familles sur leurs exploitations. En effet ces projets expliquent pour une grande part les choix, notamment techniques, faits par l'agriculteur. A partir d'indicateurs, comme la situation démographique de la famille, les exploitations de la région ont été classées selon différentes situations de l'ensemble exploitation-famille. Les stratégies d'évolution des exploitations et les actions de développement adaptées à ces stratégies ont pu ainsi être mises en évidence. Une telle démarche a mis en lumière l'importance de l'analyse au niveau de l'exploitation agricole pour l'étude de l'activité agricole. Mais cette étude a considéré les exploitations indépendamment de leur localisation dans le territoire et de leur voisinage, aussi est-il apparu indispensable, pour raisonner les problèmes d'aménagement et de développement au niveau d'une petite région, de retenir des unités restreintes de territoire dans lesquels étaient analysées, bien que sommairement, les exploitations et leurs relations entre elles. Ce niveau d'étude nous a montré l'importance de la répartition spatiale des exploitations et le rôle des résidents non agricoles dans l'évolution des exploitations (Groupe de recherches I.N.R.A.-E.N.S.S.A.A., 1977).

Ce très bref rappel des résultats des travaux précédents permet de comprendre les choix des différents niveaux d'analyse dans la recherche en Castagniccia. Cinq niveaux ont été retenus : le niveau de l'exploitation, complété par deux niveaux correspondants chacun à une échelle d'espace : le village et le massif ; le niveau représenté par les institutions et enfin celui des références écologiques, techniques et économiques.

\section{A. L'exploitation}

C'est le niveau le mieux adapté à la compréhension du choix et de l'organisation des pratiques et de l'activité agricole (OSTY, 1978) ; l'exploitation est aussi l'objet de certaines décisions relatives au développement et à l'aménagement. C'est à ce niveau que les membres de l'équipe ont le plus « d'expérience » de recherche. Mais en Castagniccia, l'exploitation apparaît très différente de celle du continent. En effet, les ćtables, les porcheries, les bergeries existent rarement ; le territoire est mal délimité ; seul le troupeau est identifiable, et encore est-il souvent difficile de connaître à un instant donné l'effectif d'un troupeau de «porcs coureurs » par exemple.

\section{B. Le village}

Plusieurs faits ont révélé l'importance de ce niveau pour notre étude. Le compartimentage géographique dû au relief, l'habitat groupé mitoyen, l'urbanisme méditerranéen où la place est un lieu de vie et d'échanges, l'organisation de 
la vie politique font des villages de la Castagniccia des unités assez autonomes. Ces villages sont des unités socio-économiques certes moins cohérentes qu'autrefois, mais encore très influentes sur le devenir de chacun, au point que nous les avons considérées comme le plus petit système agraire adapté à l'étude des activités agricoles et non agricoles. En effet, les agriculteurs et les éleveurs sont souvent minoritaires dans la population active des villages et, dans les conditions socio-économiques actuelles, l'agriculture ne peut fournir qu'un nombre insuffisant d'emplois. De plus l'imbrication d'activités agricoles avec d'autres activités est particulièrement fréquent et complexe ; ainsi 70 p. 100 des actifs non agricoles possèdent des petits élevages.

Les anciennes mises en valeur du territoire présentent un caractère de pérennité particulièrement marqué. Les cultures arbustives (vignes) ou arboricoles (fruitiers, oliviers, châtaigniers), les terrasses, les chemins, le parcellaire sont autant de traits du passé qui se répercutent sur les possibilités de production. L'utilisation actuelle du sol ne peut se comprendre sans prendre en compte ces marques contraignantes inscrites dans le cadre du village.

\section{Le massif}

Géographiquement c'est une région bien délimitée entre la mer et le massif granitique du centre de l'île, et entre les deux fleuves Golu et Tavignanu. De plus, la plaine fait partie du territoire des communes de montagne qui la bordent. Ainsi la région considérée est celle du massif et des plaines environnantes.

Historiquement, elle a été la région la plus peuplée de Corse et elle était incluse dans l'ancienne «Terra di communu » où la gestion du territoire était communautaire. Zone la plus riche en châtaigniers, elle a été aussi marquée culturellement plus que toute autre par la civilisation que cet «arbre à pain » a organisé autour de sa production.

\section{Les institutions}

Elles sont constituées des organismes publics et professionnels, des divers groupes sociaux concernés par le développement de la Castagniccia. Il est important de connaitre les divers objectifs que chacun d'entre eux poursuit, les conflits qui en résultent et leur poids respectif dans l'évolution socio-économique de la région. Au moment de la recherche il s'agissait plus précisément des syndicats de Pieve, du Mouvement de défense des exploitations familiales (MODEF), de la Fédération départementale des syndicats d'exploitants agricoles (FDSEA), de l'Union départementale des syndicats d'exploitants agricoles (UDSEA), de la Chambre d'agriculture, de la Société de mise en valeur de la Corse (SO.MI.VAC.), de coopératives, d'associations culturelles comme "A Rustagha », de l'administration (DDA, Mission interministérielle...).

\section{E. Les références élémentaires ponctuelles}

Les informations sur le milieu physique (climat, sol) et sur les diverses productions végétales (châtaignier, cultures fourragères...) ou animales (porc, chèvre...) sont apparues rares sinon inexistantes en Castagniccia, aussi a-t-il fallu mettre en place un système d'acquisition de références élémentaires indispensables à toute étude sur les potentialités agricoles.

\section{PRÉSENTATION CHRONOLOGIQUE DES ACTIONS DE RECHERCHES (tabl. 1)}

Le tableau 1 présente chronologiquement le déroulement de la recherche selon les divers niveaux. Trois types d'informations sont indiqués :

- des actions de recherches qui sont autant de sousprogrammes,

- des événements ayant eu une influence sur la recherche : changement dans la constitution de l'équipe, disponibilité de moyens nouveaux, publications ou présentation orale de certains résultats,

- des liaisons entre ces actions.

Toutes les actions et événements ne sont évidemment pas représentés sur ce schéma. Aussi est-il probable que chaque membre de l'équipe aurait construit une grille légèrement différente. Six faits plus particulièrement marquants sont commentés ci-dessous :

\section{A. Le tour de pays (mai 1974)}

Le tour de pays est une reconnaissance pluridisciplinaire de la région qui s'est déroulée au début de la recherche au niveau du massif. Aux données bibliographiques et aux analyses cartographiques s'ajoutent, au gré d'itinéraires variés, des observations et des enregistrements paysagiques et des témoignages divers. Cette démarche exploratoire, faite collectivement, parait fondamentale car ce premier contact avec un paysage, l'esprit n'étant pas guidé par le souci d'observer un aspect particulier, fournit un ensemble de questions qui, une fois organisées peuvent être à l'origine de diverses pistes de recherches et du choix des niveaux. C'est le tour de pays qui a montré l'intérêt d'une analyse au niveau des villages et de leur terroir.

\section{B. L'analyse de quelques villages (juin 1974-janvier 1975)}

L'analyse de situation de quelques villages a montré des situations démographiques tranchées: villages désertés en hiver ou présentant un noyau de population permanente et villages-centres ayant conservé une vitalité certaine. La situation foncière particulièrement complexe a été éclairée par l'analyse fine de quelques situations, notamment celle créée par une initiative des habitants d'une commune pour résoudre le problème de l'installation d'un groupe de jeunes éleveurs de porcs.

L'analyse historique et territoriale des communautés villageoises s'est avérée essentielle pour préciser les étapes et les conditions de leur évolution récente, pour comprendre que la place et les caractéristiques de l'élevage actuel n'étaient pas traditionnelles, mais qu'elles résultaient d'une adaptation récente à une situation nouvelle. Elle a permis de saisir le caractère résiduel de certaines activités, le rôle joué par les Corses du continent présents pendant l'été, l'importance de la pluriactivité et des multi-revenus au sein d'une même famille (d'où la notion de système d'activités), la situation inextricable du foncier...

C'est également au niveau du village que l'élevage s'est révélé clairement comme l'utilisateur quasi exclusif du territoire et que sont apparues l'originalité et la diversité des pratiques d'élevages. Dans quelles conditions ces pratiques étaient-elles mises en cuvre ? Cette question a orienté la recherche vers l'étude de deux élevages $(\$ D)$ puis vers la constitution d'une typologie d'élevage $(\$ \mathrm{E})$. 


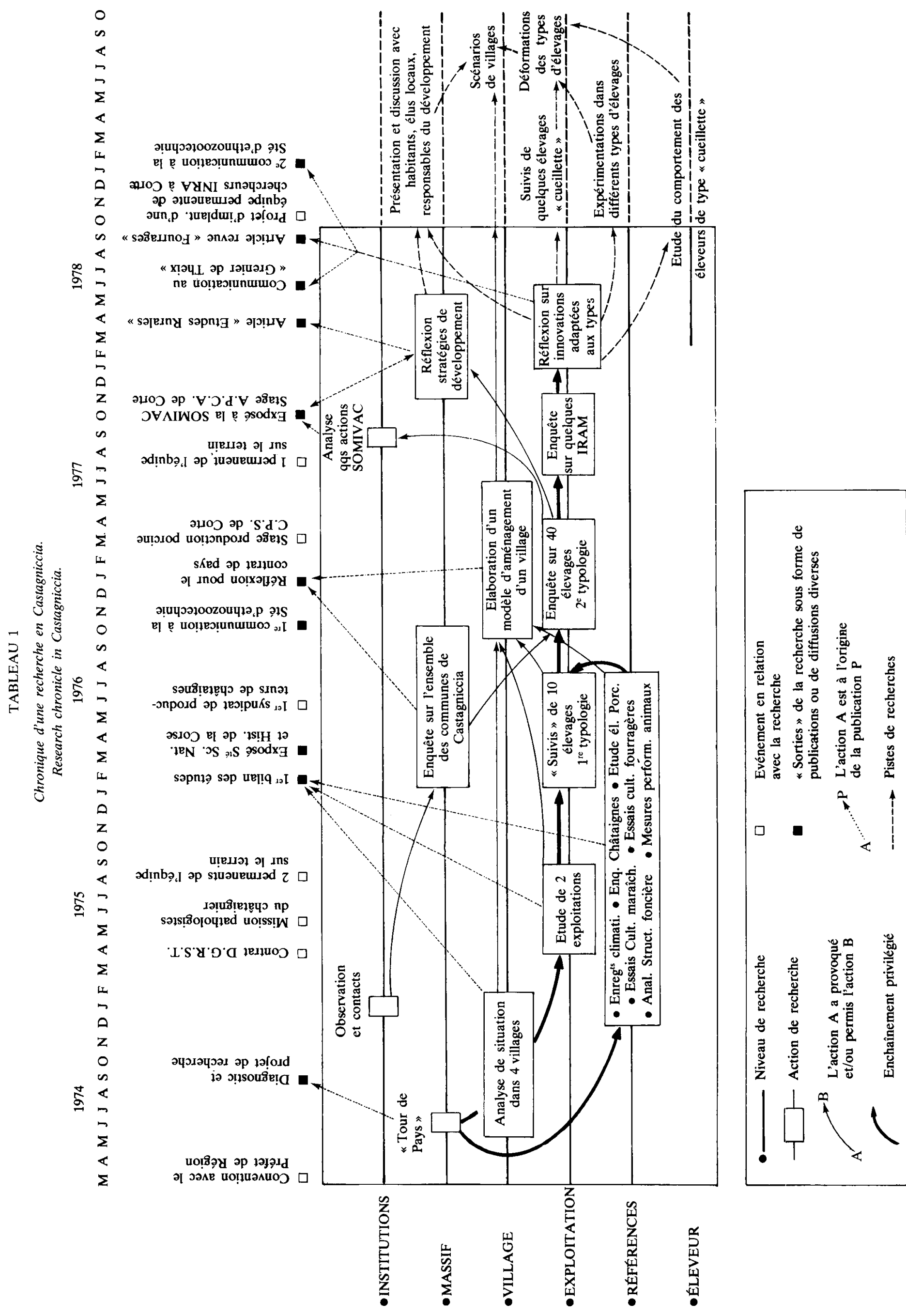


L'analyse de ces villages, puis les contacts et observations diverses faits depuis le début de l'étude ont montré l'intérêt de dégager une typologie des villages selon leur aptitude au changement. Pour cela, compte tenu de la mauvaise qualité des données statistiques disponibles, une enquête exhaustive au niveau des 111 communes de la Castagniccia a été mise en place (niveau massif). Les données de cette enquête étaient prévues comme base d'échanges avec les habitants, les élus locaux, les responsables professionnels, mais faute d'interlocuteurs intéressés ces échanges n'ont pas eu lieu.

\section{Références élémentaires (décembre 1974-octobre 1977)}

A chacune des étapes: tour de pays, analyse de situation dans quelques villages, étude de divers cas d'élevages, l'acquisition de références élémentaires, concernant le milieu physique, les productions végétales et animales, s'est avérée nécessaire. De telles références généralement disponibles dans les autres régions françaises ne l'étaient pas dans la montagne corse. Il n'existait par exemple aucun enregistrement thermométrique en Castagniccia, d'où la nécessité de compléter, même de façon partielle, les informations pluviométriques obtenues par la SO.MI.VA.C.

Pour trois productions végétales: le châtaignier, les productions fourragères et maraîchères, ont été étudiées les structures, les pratiques de production et de transformation. La production du châtaignier ayant en quelque sorte une valeur de symbole pour la population rurale corse, une association, l'"A. Rustagha ", avait déjà sensibilisé les habitants sur la nécessité de sauver les arbres existants et sur l'intérêt de la châtaigne de bouche. Mais il manquait un diagnostic précis sur les potentialités des différentes variétés et une définition des modes de traitements antiparasitaires et de conduite des arbres. Dans ce but, il a été fait appel à un pathologiste de l'I.N.R.A. et à un ingénieur de l'Institut National de Vulgarisation pour les Fruits, Légumes et Champignons (I.N.V.U.F.L.E.C.). Les résultats des travaux ont eu des répercussions larges et rapides, au moins dans un premier temps: création d'un groupement des producteurs de châtaignes, recrutement et formation d'un technicien castanéicole permanent. Il y avait dès lors matière à diffusion d'innovations techniques, mais restaient les problèmes de la place respective de la châtaigne de bouche et de l'aliment pour les porcs au niveau à la fois des exploitations et des terroirs, ainsi que celui des possibilités de conservation, de transformation et d'écoulement. A l'occasion du contrat de pays de la «petite Castagniccia», ce problème a été abordé dans le cadre d'une réflexion sur l'aménagement d'un type de village.

Les cultures maraîchères, pratiquées sous forme de jardinage et sur des petites terrasses aux alentours des villages, apparaissaient importantes comme pare-feux aux alentours des villages et comme revenu d'appoint ; mais quelles étaient les gammes de production et de techniques adaptées aux différents sites ? Les résultats des essais mis en place, pour répondre à ces questions, ont été très partiels, la principale cause étant l'absence de support professionnel pouvant prendre le relais de ces incitations.

Enfin les possibilités de ressources fourragères locales ont été étudiées. Les très intéressants essais du C.E.P.E. (ETIENNE, 1977) étant mal adaptés à des zones déclives telle que la Castagniccia, un agronome venu compléter l'équipe (septembre 1975) a mis en place des essais de comportement de plantes fourragères dont les résultats se sont avérés convergents avec le diagnostic d'un spécialiste des fourrages méditerranéens venu en Corse (CRESPO, 1977). Mais les réactions des éleveurs ayant tous apparemment besoin d'un appoint de fourrages pour l'hiver ont été très différentes. Chez l'un la culture fourragère a été exploitée, chez l'autre elle s'est dégradée, chez le troisième elle a été abandonnée dès la première année.

Pourquoi ces diverses réactions des éleveurs de Castagniccia à des propositions techniques ? Comment poursuivre la recherche sans préciser la diversité des pratiques d'élevage et sans tenter de comprendre les conditions dans lesquelles elles étaient mises en cuvre au niveau de l'exploitation?

Pour ce qui est des animaux, on a pris l'initiative de l'étude de l'élevage du porc corse (race, conduite, alimentation, production, transformation). Elle a été soutenue et approfondie par des généticiens de l'I.N.R.A. préoccupés par les possibilités d'utilisation des terrains marginaux par des races locales, selon des systèmes d'élevage extensifs adaptés.

\section{L'étude de deux élevages (mars-novembre 1975)}

L'analyse de deux élevages du village d'Ortiporio a été déterminante pour la suite de la recherche, par les questions qu'elle a soulevées :

- Pourquoi les caractéristiques structurelles de l'exploitation considérées habituellement comme très peu mobiles (surface, effectif des troupeaux, voire espèce) étaient-elles aussi variables et pourquoi les techniques de conduite des troupeaux, apparemment simples, étaient-elles aussi stables? Dans de telles conditions, comment définir une exploitation agricole en Castagniccia ?

- Quelles étaient, dans le détail, les pratiques d'élevages et quelles étaient leur « efficacité »? La méconnaissance du fonctionnement et des résultats de ces élevages a entraîné la mise en place de mesures de performances dans plusieurs élevages. Là encore, le constat a été inattendu ; il s'est avéré impossible, dans la plupart d'entre eux, d'obtenir les informations collectées habituellement dans les élevages du continent. Par exemple, la production laitière individuelle n'avait pas de signification auprès de certains éleveurs. Cette diversité de réaction des éleveurs devant la collecte de référence a suscité une typologie des élevages $(\S \mathrm{E})$.

- Compte tenu des besoins des troupeaux pendant la période d'hiver, de l'arrêt de la végétation au cours de cette saison et de la quasi-disparition de la transhumance hivernale dans la plaine orientale, comment les éleveurs ajustaient-ils les besoins et les ressources disponibles?

\section{E. La typologie des élevages (février 1976-août 1977)}

La diversité des pratiques d'élevages et des comportements des éleveurs vis-à-vis des mesures de performances ou des changements techniques proposés montrait l'intérêt de dégager une typologie des élevages de la Castagniccia. Celle-ci devenait l'axe principal de la recherche et amenait à privilégier en 1976 et 1977 le niveau de l'exploitation. Cette typologie était fondée sur le constat indiqué précédemment: les techniques adoptées par les éleveurs étaient diverses et originales et semblaient stables, alors que les structures subissaient des variations importantes. C'est donc à partir de critères techniques que s'est élaborée la typologie. Les critères retenus étaient les pratiques de mise en valeur du territoire et de conduite des troupeaux. On distinguait ainsi 6 types d'élevages caractérisés chacun par un ensemble cohérent de techniques, formant ce que nous 
avons appelé un système de pratiques. Parmi eux, deux peuvent illustrer la diversité des élevages: les systèmes de pratiques «cueillette et surveillance » et «pâturage aménagé, cueillette et conduite suivie».

Dans le système de pratiques "cueillette et surveillance », le troupeau pacage librement un ensemble non délimité de parcelles qui peut s'étendre sur une partie ou la totalité du territoire d'une ou plusieurs communes. L'éleveur n'a pas d'action directe sur le sol et la végétation. Le seul moyen de lutter contre la progression du maquis et la diminution des ressources fourragères est le feu. En conséquence, les ressources disponibles pour le troupeau sont celles que fournit la végétation au cours de son cycle annuel. L'ajustement de la production s'effectue donc au niveau des ressources des parcours pendant la période de disette. La lactation des femelles, peu alimentées, se réduit considérablement et l'éleveur évite le tarissement total en distribuant quelques bottes de foin achetées lorsque les conditions de pâturage sont trop néfastes. Il s'agit pour lui de conserver le potentiel productif des animaux. La lactation reprend au printemps lorsque la végétation naturelle des parcours fournit de nouveau des ressources fourragères importantes. Ainsi la production laitière suit étroitement le cycle végétal des parcours. L'éleveur surveille ses animaux lors des périodes de mise bas et de sevrage. Il les oriente sur les parcours, corrige leurs écarts, ayant ainsi une action plus indicatrice que directive. Les chiens ne sont utilisés que pour protéger les animaux contre les prédateurs, le renard en particulier. Les actions sanitaires, uniquement curatives, n'ont lieu que lorsque la maladie s'étend et met en cause la production du troupeau. La mortalité est donc importante. La sélection des animaux se fait sur l'aptitude de reprise de la lactation au printemps et de récupération du poids en été. Elle prend en compte également des critères de grégarité, de défense contre les ravageurs, qualités qui permettent au troupeau d'être autonome, résistant et groupé. Plus que les autres, la race Corse présente ces caractères; tous les troupeaux élevés selon ce système de pratiques sont en effet de race locale. Ces pratiques ne nécessitent pas de bâtiment aménagé. L'élevage est de plein air intégral. L'abri indispensable pour la traite n'est pas compartimenté et ne permet pas un stockage important d'aliments.

Le système «cueillette et surveillance» se caractérise donc par: une non-intervention de l'éleveur sur le territoire, une surveillance passive, des soins minimum, une alimentation hivernale juste suffisante pour poursuivre la production, et des investissements en matériel, bâtiments, clôtures, pratiquement nuls. 70 p. 100 des éleveurs de montagne mettent en œuvre ce système ou des systèmes de pratiques proches de cette description.

Dans le système de pratiques «pâturage aménagé, cueillette et conduite suivie ", le déficit alimentaire hivernal est comblé par la distribution de foin acheté ou produit par l'éleveur lui-même, et parfois par le pâturage de réserves fourragères sur pied (orge, avoine, luzerne). L'éleveur alimente ses animaux de façon à obtenir une production plus régulière, moins sensible aux aléas climatiques et aux saisons. Il les surveille et les aide efficacement lors de la mise bas et du sevrage et essaie de prévenir les maladies. Il a une action directive sur les déplacements, surtout sur les parties aménagées. Il guide son troupeau. La production individuelle des animaux est importante et la sélection est orientée en ce sens. Des croisements avec des animaux plus productifs sont pratiqués (brebis sardes, chèvres alpines). Les abris et bâtiments possèdent des aménagements intérieurs permettant l'allotement du troupeau, la mise à l'écart des animaux malades et le stockage des réserves hivernales.
Ce système de pratiques associant la culture et la nonculture se caractérise par une conduite suivie du troupeau, des soins préventifs, une recherche de la production individuelle. En contrepartie, les charges, les investissements, sont considérables par rapport à ceux décrits précédemment : 30 p. 100 des éleveurs de montagne utilisent ce système de pratiques ou un système qui lui est proche.

Cette typologie a été testée sur un échantillon aléatoire de 31 éleveurs tirés dans la population des 410 éleveurs de l'ensemble du massif. C'est auprès de ceux-ci qu'ont été analysées les conditions du choix de ces systèmes de pratiques, en prenant en compte les données structurelles des exploitations telles que la situation géographique, la constitution démographique de la famille, la formation des éleveurs, l'appartenance à un clan.

Le problème majeur soulevé par la typologie et par les contacts avec plusieurs institutions du développement de l'agriculture est l'absence ou l'inadéquation des actions de développement auprès de la majorité des éleveurs de la Castagniccia.

La question principale que pose la présence des différents types d'élevage au niveau des exploitations est: quels changements pour quels élevages? Notamment, quelles innovations pour les éleveurs ayant opté pour des systèmes de pratiques de type cueillette et surveillance et pour lesquelles les propositions actuelles sont soit sans effet à terme, soit entraînent une modification fondamentale du système d'élevage ? Quelle peut être la structure du conseil technique auprès de ces éleveurs ? On conçoit la nécessité d'une recherche de références écologiques, techniques, économiques adaptées à ces élevages. Un programme de «suivis » ou d'observations programmées est nécessaire, notamment sur l'ensemble constitué par le troupeau et le couvert végétal. Pour cela, il faut définir des méthodes d'analyses nouvelles, car comment caractériser le sens et le rythme de l'évolution d'un couvert végétal complexe, composé de ligneux, de semi-ligneux et d'herbe, sous l'influence d'un système d'élevage de type cueillette? La question est fondamentale, car s'il y a dégradation du couvert, on est en présence de systèmes plus ou moins éphémères; s'il n'y a pas dégradation ou dégradation très lente, ces systèmes représentent des formes originales d'utilisation de territoires marginaux, peut-être intéressantes pour des zones du Bassin méditerranéen en voie d'abandon. La question posée aux spécialistes de la dynamique de la végétation est nouvelle, car il ne peut être question ici d'utiliser le critère habituel du chargement en animaux à l'hectare; de plus il convient de mettre au point une méthode de diagnostic suffisamment rapide.

Une autre question importante provient de l'existence de ces types d'élevage dont le maintien ou la disparition apparaît déterminant pour l'horizon d'activités agricoles de la Castagniccia : qui sont ces éleveurs ? quelles perceptions ont-ils de leur situation? Pour tenter de répondre à cette question, une collaboration avec une discipline anthropologique est nécessaire.

A ce stade d'avancement de la recherche et au niveau du massif, une réflexion peut être engagée sur la question des diverses possibilités de développement des activités d'élevage. En considérant les conditions socio-politiques actuelles, nous envisageons schématiquement deux stratégies : la première consiste à imaginer une évolution spécifique à chaque type d'élevage existant et à créer les formes d'intervention permettant notamment une évolution propre des systèmes dits de cueillette, la seconde dans laquelle seuls des systèmes plus intensifs se maintiennent. C'est par 
un article publié dans la revue Etudes Rurales que nous avons voulu amorcer ce débat (CRISTOFINI et al., 1978).

Dans quelles mesures les diverses institutions concernées par le développement prennent-elles en compte la diversité des types d'élevages? (Seul l'impact des I.R.A.M. (Intervention pour la Renaissance Agricole de la Montagne) de la SO.MI.VA.C. sur les divers types d'éleveurs a été étudié). Quelles conséquences peut avoir cette analyse sur les programmes et les stratégies de ces organismes? Pour lancer une discussion sur ce thème avec les interlocuteurs et organismes intéressés, l'équipe de recherche a monté un film (*) sur le travail d'un berger de Castagniccia. Par là, c'est aussi un essai de diffusion des résultats d'une recherche interdisciplinaire qui est testé.

\section{LES PRINCIPAUX RÉSULTATS DE LA RECHERCHE}

Outre les résultats méthodologiques $(\S \mathrm{VI})$, les retombées d'une telle recherche sont diverses, s'échelonnent au cours de son déroulement et peuvent même donner lieu à des réalisations longtemps après sa conclusion.

En premier lieu, certains traits généraux de l'agriculture de la Castagniccia ont été soulignés : climat d'abandon, malgré quelques signes de renouveau, combinaisons d'activités au niveau familial, empirisme des techniques et absence d'encadrement technique et d'organisation économique des agriculteurs, rareté des groupes, des associations et des coopérations intervillageoises, conflits entre éleveurs et propriétaires, pouvoir important détenu par les élus locaux.

Par ailleurs, la recherche révèle un fort potentiel de production (gammes et niveaux) et met en évidence une diversité des activités agricoles plus grande que ne le laissait prévoir une observation rapide. Cette adaptation aux contraintes multiples et souvent nouvelles, plus particulièrement des éleveurs, est un aspect des potentialités car elle montre à la fois une résistance et une adaptation de la part de certains habitants. Le fort potentiel et la manifestation, même limitée, de vitalité rend plausible un développement de cette région et justifie des actions dans ce but.

Parmi les retombées locales ou régionales, il faut signaler les résultats sectoriels concernant la châtaigneraie. Ainsi, avec l'aide de spécialistes, le potentiel de la châtaigneraie a été précisé par reconnaissance des diverses variétés, par la caractérisation de leurs aptitudes et la définition des techniques adaptées de régénération. Ces données ont fourni une aide à la formation de groupements de producteurs et ont permis d'obtenir des moyens financiers du Comité National Interprofessionnel de la Châtaigne et du Marron (C.N.I.C.M.). Elles ont servi aussi de programme de travail, en particulier pour un conseiller castanéicole permanent. Il n'en reste pas moins que des travaux restent à faire, notamment pour la délimitation des zones, dont l'une d'elles devrait être réservée à la châtaigne de bouche, et pour la conservation, la transformation et l'écoulement des fruits.

Pour les productions fourragères, des essais ont montré l'importance du potentiel fourrager en dessous de $800 \mathrm{~m}$ et l'intérêt de certaines espèces, fétuque et trèfle souterrain en particulier, pour combler le déficit hivernal. Un thème

(*) Disponible à l'U.R.S.A.D. (Unité de Recherches sur les Systèmes agraires et le développement) du C.N.R.A., Route de StCyr, 78000 Versailles). primordial s'est dégagé : celui du maintien, voire du développement des surfaces herbacées dans des terrains où, actuellement, le maquis gagne, en utilisant les animaux euxmêmes pour éviter l'usage systématique du feu lorsque les conditions de milieu empêchent toute intervention mécanique. Sur le plan des productions animales, certaines particularités des races locales et de diverses pratiques utilisées ont été précisées, mais des analyses complémentaires s'imposent.

Un résultat important est celui de la caractérisation des divers types d'élevages qui s'avèrent très variés et originaux. Leur mise en cuvre se fait dans le cadre de situations foncières, familiales, sociales diverses. Ainsi se dégagent des systèmes de pratiques d'élevages différents qui s'inscrivent dans des formes de productions particulières. $\mathrm{Ce}$ résultat a servi de base de réflexion à un stage organisé au Centre de Promotion Sociale (C.P.S.) de Corte par les services de formation de l'A.P.C.A., le centre de formation d'Etchary et l'équipe de recherche pour les conseillers et techniciens des deux départements de l'île, sur le thème de la connaissance du milieu. Il a fait également l'objet de séances de travail avec des responsables de la SO.MI.VAC. La connaissance de ces élevages permet de formuler en termes mieux adaptés le problème des recherches des références au niveau des productions animales et suggère des protocoles de «suivis » de troupeaux, étape indispensable à l'élaboration de propositions techniques.

Plusieurs remarques permettent de penser que les types d'élevage mis en évidence ne sont pas le propre de la Castagniccia, mais d'une grande partie de l'intérieur de l'île. En effet, les quelques systèmes observés hors de la Castagniccia pouvaient clairement se rattacher à l'un ou l'autre des types définis; de plus, des conseillers agricoles travaillant dans diverses régions ont confirmé ce point de vue. La mise en évidence de divers types d'élevages extensifs dans la montagne Corse et de leur fonctionnement a un intérêt qui dépasse la Corse, car elle fournit des éléments de réflexion pour des stratégies alternatives de développement dans les zones montagneuses du Bassin méditerranéen soumises à un exode rural avancé.

Il convient d'ajouter à ce bref rappel des résultats, celui portant sur quelques concepts qui sont à préciser et à discuter mais qui, d'ores et déjà, paraissent présenter un intérêt pour l'analyse du développement. Le premier est celui des pratiques dont la compréhension, par opposition aux techniques, n'est pas possible sans la connaissance de celui qui agit et des conditions dans lesquelles il se situe. Le second concept, issu du premier, est celui de systèmes de pratiques: ensemble de pratiques liées entre elles et qui est caractéristique d'une stratégie globale de l'agriculteur. Il y a enfin la notion de formes de production. Chacune d'elles comprend un ensemble de traits socio-économiques et du milieu physique liés entre eux, ensemble, dans lequel s'inscrit de façon apparemment cohérente un système de pratiques.

\section{RÉFLEXIONS MÉTHODOLOGIQUES}

La présentation de cette chronique est faite dans un souci de recherche sur la recherche. Elle est proposée à d'autres équipes comme une expérience parmi d'autres et est soumise à la critique. Cependant, dans le but de dégager des éléments d'une trame méthodologique applicable à ce type de recherches, plusieurs réflexions peuvent être faites.

$\mathrm{Au}$ préalable on peut tenter de caractériser de façon schématique ce type de recherche. Elles présentent un niveau 
d'intégration élevé en ce sens qu'elles prennent en compte des facteurs du milieu physique, mais aussi des variables représentatives des activités humaines et de l'organisation sociale. Ce caractère global entraîne un risque permanent de dispersion, de variation d'objectif, de boursouflement de la recherche. Elles sont en outre plus ou moins liées aux actions de transformation du milieu et notamment aux projets de développement ; elles posent de ce fait continuellement le problème des relations avec les «acteurs" dont les objectifs peuvent être conflictuels entre eux et avec ceux des chercheurs.

Au départ de la recherche, compte tenu de la ou des questions que l'on se pose ou qui sont posées, une phase préalable de réflexion et d'observation est nécessaire pour savoir comment aborder concrètement l'étude : analyse de la demande, analyse de situation. Peut-on systématiser cette étape exploratoire devant déboucher sur un diagnostic de recherche et au cours de laquelle les méthodes qualitatives sont particulièrement importantes?

Un problème majeur est celui du choix des niveaux de recherche adaptés aux objectifs et, pour chacun d'eux, des échelles de temps pertinentes. En Castagniccia, cinq niveaux avaient été retenus au départ, mais au cours de la recherche il y a eu réduction progressive de son objet qui s'est traduit par une concentration des efforts sur un niveau d'analyse particulier, celui de l'exploitation agricole. Ce n'est qu'après l'investigation faite à ce niveau qu'il y a eu élargissement du champ de la recherche. Un niveau non prévu au départ a d'ailleurs été introduit dans cette phase d'élargissement, celui du comportement de l'éleveur. On peut penser qu'à chaque objectif correspond un niveau d'analyse privilégié.

D'une façon générale se pose la question des relations entre différents niveaux de recherche, que ce soit le passage d'une unité englobante à une unité englobée et inversement, ou que ce soit la mise à jour de phénomènes à un niveau au cours d'une analyse faite à un autre niveau. La recherche de références techniques permet d'illustrer ce dernier point. En effet, les essais de diverses plantes fourragères, par exemple, ont induit des réactions très marquées des éleveurs qui remettaient en cause le diagnostic des techniciens ou du moins sa généralité. De même, l'impossibilité d'obtenir des mesures de production ou de performances animales chez les éleveurs ne cadrait pas avec le schéma technique habituel. Admettant que cette disparité de réactions avait des causes profondes chez les éleveurs, la recherche de références techniques jouait alors un rôle moteur dans la progression des travaux au niveau des exploitations.

Par ailleurs, on est contraint de considérer des échelles de temps différentes sclon les cycles biologiques et selon les rythmes d'évolution des faits socio-économiques pris en compte, ce qui rend difficile une programmation.

Malgré ces difficultés, il paraît utile de réaliser une esquisse de recherche précédant l'étude en découpant des portions de réalités apparemment cohérentes et en s'aidant, si nécessaire, de l'analyse de système.

Un autre problème concerne la coordination chronologique des différentes actions de recherche. A ce sujet, on peut d'abord remarquer que des chercheurs avaient acquis, avant nous, dans des milieux semblables à la Castagniccia, une vaste connaissance sur le fonctionnement du maquis comme formation végétale (Programme «Tavignanu» suscité par le Comité "Gestion des Ressources Naturelles Renouvelables » de la D.G.R.S.T.) et pourtant, il nous a été impossible au début de notre recherche d'intégrer leurs résultats.
Au stade actuel de notre travail, les questions que nous posons aux biologistes sur le maquis sont nombreuses et précises, mais ils ne sont plus là et leurs résultats ne répondent pas à nos questions (tabl. 1). On peut constater par ailleurs que certaines séquences de travail se sont enchaînées dans de bonnes conditions. C'est le cas par exemple de l'enchaînement : tour de pays, analyses monographiques de villages et de quelques élevages, recherche de références sur les productions et "suivis " de plusieurs exploitations. Par contre, l'enquête menée dans les communes du massif, travail long et coûteux, a été engagée à contretemps; les types d'élevage étant insuffisamment connus, les questions à leur sujet se sont avérées insuffisantes et les réponses peu utilisables.

A ce problème peut être rattaché celui de la constitution et $d u$ fonctionnement $d u$ groupe de recherches forcément interdisciplinaire. Une difficulté provient de ce que, à certains moments, telle ou telle discipline ou tel ou tel type de chercheur (généraliste ou spécialiste) est appelé à jouer un rôle clé et que, par ailleurs, une continuité dans la progression de la recherche et une présence permanente sur lc «terrain" est indispensable. A ce sujet, nous sommes persuadés de l'intérêt de la collaboration entre des chercheurs formant un noyau permanent avec d'autres dont les interventions sont plus limitées dans le temps. Les premiers peuvent assurer la continuité et la permanence indiquées précédemment, mais aussi l'évolution plus générale d'une problématique, des concepts et des méthodes spécifiques à ce type de recherches.

Sans entrer dans les multiples problèmes posés par l'interdisciplinarité, nous pensons nécessaire d'introduire de façon plus systématique dans le champ de ces recherches une observation du groupe en recherche par lui-même. Ceci est d'autant plus important que ces travaux se déroulent sur plusieurs années et qu'ils impliquent des relations diverses avec les habitants et les institutions d'un lieu ou d'une région. Une autre constatation d'ordre chronologique concerne les «sorties » de la recherche (tabl. 1). Il n'y a pas un résultat mais des «sorties" partielles qui s'échelonnent au cours de la recherche selon des formes variées (publications, communications, stages...) et destinées à des utilisateurs différents. Les choix des cibles successives peuvent avoir des conséquences importantes sur la recherche ellemême. Ceci pose le problème très général des relations cntre les chercheurs et les « acteurs ", et celui des limites de leurs engagements tant au niveau des interventions ellesmêmes que de la formation.

Enfin nous avons constaté, à plusicurs reprises, que certaines questions jugées initialement prioritaires devenaient secondaires ou impliquaient une nouvelle formulation. On peut citer, à titre d'exemple, les structures foncières qui étaient apparues, dans une première phase de la recherche, comme un facteur de blocage déterminant pour le développement. La question qui en découlait était : comment lever les contraintes dues au foncier? Au cours de l'étude des divers élevages, on a observé que leurs situations foncières très variées paraissaient cohérentes avec le fonctionnement de chacun des systèmes d'élevage. Dès lors, la question de départ devenait celle de l'évolution des systèmes eux-mêmes. Ainsi les objectifs sont à reconsidérer au fur et à mesure des résultats. Dans ces conditions, on conçoit la nécessité d'une sorte de «pilotage à vue » de la recherche. Mais il convient, plus que dans d'autres recherches, de porter un jugement critique sur ce que l'on fait; sans évaluations critiques fréquentes, on aboutit à des travaux «sur » ou «à propos de » qui s'éloignent de la perspective 
de développement dans laquelle se situe ce type de recherches.

L'établissement d'une chronique de la recherche nous semble un moyen d'aborder divers problèmes en relation avec son déroulement. Elle peut être un outil de travail interdisciplinaire dans la mesure où elle aide à la définition des enchaînements et séquences de recherches et à la discussion de leur logique, où elle sert de support à des évaluations critiques et où elle facilite le rapprochement avec d'autres recherches.

VII. CHRONOLOGIE DES PRINCIPAUX DOCUMENTS, COMMUNICATIONS ET PUBLICATIONS CONCERNANT L'ÉTUDE ENTREPRISE

Mars 1974 - Convention de recherche avec le préfet de la région Corse : "Etude-diagnostic d'exploitations agricoles ». I.N.R.A.S.E.I., $5 \mathrm{p}$.

Nov. 1974 - Brugere D., De Casabianca F., DefFonTAINES J. P., Houdard Y., RaICHON C. Situation de l'Agriculture dans la montagne Corse. Premières réflexions. Projets de recherche. I.N.R.A.-S.E.I., 33 p.

Dec. 1974 - BRUGERE D. Bibliographie générale de la Corse. Eléments sélectionnés. I.N.R.A.S.E.I., $19 \mathrm{p}$.

Sept. 1975 - Demande d'aide à la recherche à la D.G.R.S.T. I.N.R.A.-S.E.I., 6 p.

Fev. 1976 - Brugere D., Bruno J.F., De CASA BIANCA F., DEFFONTAINES J. P., HOU DARD Y., Moisan H., Prodhomme J. P., Raichon C., De Verneuil B. Premier bilan des études entreprises dans le massif de la Castagniccia (Corse) I.N.R.A.-S.E.I., 83 p.

Avril 1976 - RAICHON C. Activités agricoles et problèmes de développement régional, un village de la Castagniccia: Ortiporio. Bull. Soc. Sci. hist. nat. Corse : 619, 43-59.

Fev. 1976 - De CASABIANCA F. Les châtaigniers corses seront-ils sauvés? I.N.R.A.-S.E.I., 4 p.

Mars 1976 - RAICHON C. Existe-t-il des solutions aux problèmes fonciers de la Castagniccia ? L'antagonisme propriétaire foncier-exploi- tant agricole est-il irréductible ? I.N.R.A.S.E.I., 12 p.

Nov. 1976 - Molenat M., Raichon C., De Verneuil B. L'élevage du porc et la charcuterie en Castagniccia. Ethnozootechnie 16, 68-86.

Juin 1976 - De VERnEuIL B. Les espèces animales face au développement de la montagne Corse : I.N.R.A.-S.E.I., $3 \mathrm{p}$.

Jan. 1977 - Brugere D., DefFontaines J. P., RaICHON C., De Verneuil B. Réflexions sur le développement des activités agricoles à l'occasion du contrat de pays de la Castagniccia. I.N.R.A.-S.E.I. 21 p.

Avril 1977 - De CASABIANCA F. Châtaigneraie et développement de la Corse, réflexions à partir de la Castagniccia. I.N.R.A.-S.E.I., 18 p.

Avril 1978 - Cristofini B., DefFontaines J. P., RAIChON C., De Verneuil B. Pratiques d'élevage en Castagniccia. Pour une exploitation du milieu naturel et social. Etudes rurales, 71-72, 89-109.

Juin 1978 - Deffontaines J. P., Raichon C., De VERNEUIL B. Utilisation des parcours par les bergers de la Castagniccia. Conséquences pour la recherche et le développement. Utilisation par les ruminants des pâturages d'altitude et parcours méditerranéens. $X^{e}$ Journée du Grenier de Theix, I.N.R.A., $1979,409-425$.

Août 1978 - Defrontaines J. P., Raichon C. De VerNEUIL B. L'amélioration de la production fourragère en Corse intéresse-t-elle les éleveurs? Fourrages 75, 3-28.

Dec. 1978 - Deffontaines J. P., Raichon C. De VerNEU1L B. Pratiques d'élevage et races en Castagniccia. Ethnozootechnie 24 : 41-52.

Oct. 1980 - DefFontaines J. P., RAIChon C. Systèmes de pratiques et terroir: moyens d'analyse d'une agriculture régionale ? Economie rurale, 142,30 .

Sept. 1981 - DefFontaines J. P., Raichon C. Rousso R., De Verneuil B. Auguste, berger corse : exemple d'un système de pratiques d'élevage. Film Super $8 \mathrm{~mm}$. $40 \mathrm{mn}$.

Reçu le 19 octobre 1981 Accepté le 12 novembre 1981.

\section{RÉFÉRENCES BIBLIOGRAPHIQUES}

Crespo D. G., 1977. Quelques aspects de l'amélioration des productions pastorales et fourragères en Corse. I.N.R.A., $33 \mathrm{p}$.

Cristofini B., Deffontaines J. P., Raichon C., De Verneuil B., 1978. Pratiques d'élevage en Castagniccia. Pour une exploitation du milieu naturel et social. Etudes rurales, 71-72, 89-109.

Etienne M., 1977. Un essai d'amélioration des ressources pastorales de la végétation spontanée en Corse. Fourrages, 71, 83-92.
Groupe de Recherches I.N.R.A.-E.N.S.S.A.A., 1973. Conditions du choix des techniques de production et évolution des exploitations agricoles. S.E.I. B4., 160 p.

Groupe de recherches I.N.R.A.-E.N.S.S.A.A., 1977. Pays, paysans, paysages dans les Vosges du Sud. I.N.R.A., $192 \mathrm{p}$.

Osty P. L., 1978. L'exploitation vue comme un système. Diffusion de l'innovation et contribution au développement. Bull. tech. Inf. Min. Agric., 326, 43-49. 